$\mathrm{PET} / \mathrm{CT}$ in lung cancer

\title{
Mid-radiotherapy PET/CT for prognostication and detection of early progression in patients with stage III non-small cell lung cancer
}

\author{
Michael F. Gensheimer ${ }^{\mathrm{a}, \mathrm{b}, *, 1}$, Julian C. Hong ${ }^{\mathrm{a}, \mathrm{c}, 1}$, Christine Chang-Halpenny ${ }^{\mathrm{a}, \mathrm{d}}$, Hui Zhu ${ }^{\mathrm{a}, \mathrm{e}}$, \\ Neville C.W. Eclov ${ }^{\mathrm{a}, \mathrm{f}}$, Jacqueline To ${ }^{\mathrm{a}, \mathrm{g}}$, James D. Murphy ${ }^{\mathrm{a}, \mathrm{h}}$, Heather A. Wakelee ${ }^{\mathrm{b}, \mathrm{i}}$, Joel W. Neal ${ }^{\mathrm{b}, \mathrm{i}}$, \\ Quynh-Thu Le ${ }^{\mathrm{a}, \mathrm{b}}$, Wendy Y. Hara ${ }^{\mathrm{a}, \mathrm{b}}$, Andrew Quon ${ }^{\mathrm{j}}$, Peter G. Maxim ${ }^{\mathrm{a}, \mathrm{b}}$, Edward E. Graves ${ }^{\mathrm{a}, \mathrm{b}}$, \\ Michael R. Olson $^{\mathrm{a}, \mathrm{k}}$, Maximilian Diehn ${ }^{\mathrm{a}, \mathrm{b}, \mathrm{l}, *}$, Billy W. Loo Jr. ${ }^{\mathrm{a}, \mathrm{b}, *}$
}

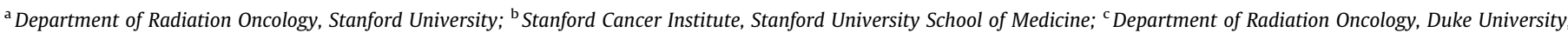

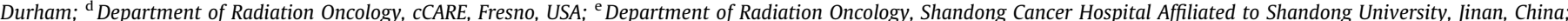

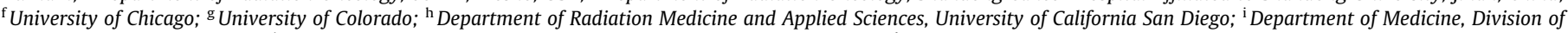

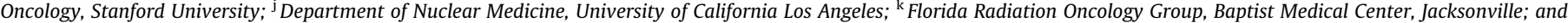
${ }^{1}$ Institute for Stem Cell Biology and Regenerative Medicine, Stanford University, USA

\section{A R T I C L E I N F O}

\section{Article history:}

Received 5 November 2016

Received in revised form 22 May 2017

Accepted 5 August 2017

Available online 19 August 2017

\section{Keywords:}

Non-small cell lung cancer

FDG PET

Biomarkers

Radiation therapy

\begin{abstract}
A B S T R A C T
Background and purpose: Pre- and mid-radiotherapy FDG-PET metrics have been proposed as biomarkers of recurrence and survival in patients treated for stage III non-small cell lung cancer. We evaluated these metrics in patients treated with definitive radiation therapy (RT). We also evaluated outcomes after progression on mid-radiotherapy PET/CT.

Material and methods: Seventy-seven patients treated with RT with or without chemotherapy were included in this retrospective study. Primary tumor and involved nodes were delineated. PET metrics

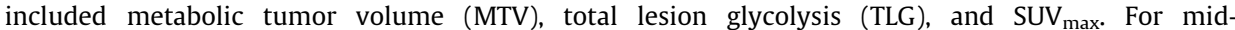
radiotherapy PET, both absolute value of these metrics and percentage decrease were analyzed. The influence of PET metrics on time to death, local recurrence, and regional/distant recurrence was assessed using Cox regression.

Results: $91 \%$ of patients had concurrent chemotherapy. Median follow-up was 14 months. None of the PET metrics were associated with overall survival. Several were positively associated with local recurrence: pre-radiotherapy MTV, and mid-radiotherapy MTV and TLG $(p=0.03-0.05)$. Ratio of mid- to pre-treatment SUV $_{\max }$ was associated with regional/distant recurrence $(p=0.02)$. 5/77 midradiotherapy scans showed early out-of-field progression. All of these patients died.

Conclusions: Several PET metrics were associated with risk of recurrence. Progression on midradiotherapy $\mathrm{PET} / \mathrm{CT}$ was a poor prognostic factor.
\end{abstract}

ㄷ 2017 Elsevier B.V. All rights reserved. Radiotherapy and Oncology 125 (2017) 338-343
Patients with unresectable stage III non-small cell lung cancer (NSCLC) are generally treated with 6-7 weeks of definitive concurrent chemoradiotherapy (chemoRT), and the option of additional consolidation chemotherapy. These patients have poor prognosis, with median overall survival of 10-14 months historically [1], although more favorable median survival of over 20 months is seen in patients with good performance status and modern staging [2]. Biomarkers that indicate which patients are likely to suffer local or distant recurrence could be used to tailor treatment type and

* Corresponding authors at: 875 Blake Wilbur Dr. MC 5847, Stanford, CA 94305, USA.

E-mail addresses: mgens@stanford.edu (M.F. Gensheimer),diehn@stanford.edu (M. Diehn), bwloo@stanford.edu (B.W. Loo Jr.).

${ }^{1}$ Co-first authors. intensity. Fluoro-2-deoxy-D-glucose positron emission tomography (FDG PET) imaging has been shown to be useful for delineation of radiation targets for stage III NSCLC [3-5], and intensity of FDG uptake is known to vary during the course of radiation therapy and/or chemotherapy [6-7]. Several retrospective studies have suggested mid-radiotherapy (mid-RT) FDG PET metrics as prognostic imaging biomarkers [8-12], but larger and ultimately prospective studies are needed to validate the concept.

There are several examples of the success of mid- or posttreatment FDG PET to determine response to cancer treatment and help tailor therapy [13]. Two recent trials in Hodgkin lymphoma demonstrated that patients with complete metabolic response on mid-chemotherapy or post-chemotherapy PET have good prognosis and may be able to undergo less intensive treatment $[14,15]$. In another trial, patients with locally advanced head 
and neck cancer were randomized to planned neck dissection after radiation therapy, or neck dissection only if post-radiation therapy PET showed residual uptake in lymph nodes [16]. Disease control was equivalent in the two groups, supporting the prognostic value of post-treatment PET. Finally, a prospective trial in patients treated with chemoRT for stage III NSCLC showed a negative association between post-treatment peak standardized uptake value and overall survival [17].

We sought to validate the prognostic value of mid-RT FDG PET metrics in patients with stage III NSCLC with the largest series reported to date. We also identified a number of patients with early disease progression on mid-RT PET/CT, and tried to gain insights into how these patients should be managed.

\section{Materials and methods}

\section{Patient population and treatment}

This retrospective study was approved by our Institutional Review Board. Patients were identified through a prospectively maintained departmental database that includes all lung cancer patients. Consecutive patients with stage III NSCLC treated with definitive radiation therapy (RT), who had pre- and mid-RT PET/ $\mathrm{CT}$, were included. This patient population routinely received mid-RT PET/CT during this time period, and mid-RT PET/CT was generally obtained around half-way through RT. Patients who received concurrent chemotherapy were included, but patients who had induction chemotherapy or surgical resection were excluded. Standard treatment at our center consists of 6-7 weeks of radiation therapy with concurrent platinum doublet chemotherapy, most commonly with weekly carboplatin and paclitaxel. Consistent RT technique was used as follows: Gross tumor volume (GTV) was defined based on imaging at simulation comprising thin-slice CT (1.25 mm slice thickness) and FDG PET/CT acquired in the treatment position, and included the primary tumor and involved regional nodes with abnormal radiological characteristics (either CT or PET). The clinical target volume (CTV) included the GTV with no further explicit expansion for microscopic extension and no inclusion of uninvolved elective nodal stations, considering the modest dose intensification already built into the prescription. An internal target volume (ITV) accounting for respiratory motion of the CTV was constructed based on 4-D CT. Based on our institutional image-guidance strategies, the final planning target volume (PTV) was constructed using a $0.5 \mathrm{~cm}$ expansion from the ITV. Almost all patients received highly conformal intensitymodulated radiation therapy (IMRT) using either multiple fixed fields (typically 6-7) or volumetric modulated arc therapy (VMAT), and 6 MV photons. Plans were normalized such that 95\% of the PTV was covered by the prescription dose. Daily pre-treatment imaging using orthogonal planar $\mathrm{kV}$ on-board imaging and at least weekly imaging with cone beam CT (CBCT) was performed with corresponding position correction prior to delivery of each fraction.

\section{PET analysis}

Most pre-RT PET/CT and all mid-RT PET/CT scans were performed on one of two scanners in our department. Before 2013, all scans were done on a GE Discovery scanner (GE Medical Systems, Milwaukee, WI). After that, most scans were done on a Siemens Biograph mCT scanner (Siemens, Erlangen, Germany). Each patient fasted for at least $8 \mathrm{~h}$ before imaging. After ensuring that blood glucose was $<180 \mathrm{mg} / \mathrm{dL}$, patients were injected with 12 $18 \mathrm{mCi}$ of FDG. After a tracer uptake time of 45-60 min, patients underwent PET/CT imaging. The PET data were reconstructed with either an ordered set expectation maximization algorithm (GE scanner), or point-spread function modeling with time-of-flight reconstruction (Siemens scanner).

For the retrospective analysis, all primary tumors and involved nodes were delineated on pre-RT and mid-RT PET using PET Edge, a gradient-based method included in MIM software (Mim Software Inc., Cleveland, $\mathrm{OH}, \mathrm{USA}$ ), consistently by a single thoracic radiation oncologist (MG). This algorithm places the contour boundary at the location where the signal gradient is highest, and has been found to correspond better with pathological specimens than thresholdbased methods [18]. In rare situations when the automatically generated contours were clearly inaccurate due to extension into uninvolved adjacent tissues, they were manually edited. The PET metrics recorded were metabolic tumor volume (MTV), total lesion glycolysis (TLG), and maximum SUV (SUV $\left.{ }_{\max }\right)$ [13]. MTV represents the volume in $\mathrm{mL}$ of metabolically active cancer. TLG is MTV multiplied by average SUV within the volume, and represents the total metabolic activity of the visible cancer. For mid-RT PET, both absolute value of these metrics and percentage change from pre-RT values were examined. The distributions of pre-RT PET metrics and absolute value of mid-RT PET metrics were highly right-skewed, so these values were log transformed prior to analysis. The influence of PET metrics on time to local recurrence, regional/distant recurrence, and overall survival was assessed using the Cox proportional hazards model. In-field local recurrence was defined as recurrence within the PTV. Out-of-field regional recurrence was defined as recurrence outside the PTV but within the same lobe of the lungs, or in regional nodes. All other recurrences were considered distant. Time to events was measured from first day of radiation therapy.

Patients with early disease progression with new lesions noted on mid-RT PET/CT were included in the prognosis analysis for preRT PET/CT, but not for mid-RT PET/CT. This is because these patients had already suffered progression at time of mid-RT imaging. Their outcomes were analyzed separately in a descriptive fashion due to the small number of early progressions.

$R$ version 3.3.2 was used for statistical analysis. For cumulative incidence plots of local and regional/distant recurrence, death was treated as a competing risk. The Karnofsky performance status was assessed at the consultation visit just before beginning RT.

\section{Results}

Seventy-seven patients treated between 2006 and 2015 were included. Patient characteristics are shown in Table 1. Ninety-one percent had concurrent chemotherapy. Median radiation therapy dose was $66 \mathrm{~Gy}$ in $2 \mathrm{~Gy}$ fractions (dose range 60-80.4 Gy, interquartile range 66-70 Gy). Mid-RT PET was obtained approximately half-way through radiation therapy: median dose at time of mid-RT PET was $34 \mathrm{~Gy}$ (range 22-48.4 Gy, interquartile range 31.8-36 Gy).

Median pre-RT MTV was $40.3 \mathrm{~mL}$ (range 0.79-597.7), median pre-RT TLG was 298 (range 3.4-4244.0), and median pre-RT $\mathrm{SUV}_{\max }$ was 15.6 (range 2.7-59.8). Five of 77 patients had early disease progression with new lesions on mid-RT PET/CT. For the other 72 patients, PET metrics generally decreased on mid-RT imaging compared to pre-RT imaging. For these 72 patients, median midRT MTV was $24.4 \mathrm{~mL}$ (range 0.02-500.0), median mid-RT TLG was 104.4 (range $0.07-2050.0$ ), and median mid-RT SUV $\max$ was 8.3 (range 1.2-38.1). Median per-patient MTV decrease was 32\%, median TLG decrease was $65 \%$, and median SUV $_{\text {max }}$ decrease was $46 \%$.

Median follow-up was 14 months (range 2-107). Median overall survival was 23 months; two-year overall survival was $46 \%$. Cumulative incidence of local recurrence at one and two years was $18 \%$ and $24 \%$, respectively; cumulative incidence of regional/ 
Table 1

Patient characteristics.

\begin{tabular}{|c|c|}
\hline Characteristic & Median (range) or number \\
\hline Age at treatment & $68(42-90)$ \\
\hline \multicolumn{2}{|l|}{ Sex } \\
\hline Female & $26(34 \%)$ \\
\hline Male & $51(66 \%)$ \\
\hline \multicolumn{2}{|l|}{ Karnofsky performance status } \\
\hline 100 & $6(8 \%)$ \\
\hline 90 & $16(21 \%)$ \\
\hline 80 & $34(44 \%)$ \\
\hline 70 & $15(19 \%)$ \\
\hline 60 & $4(5 \%)$ \\
\hline 50 & $2(3 \%)$ \\
\hline \multicolumn{2}{|l|}{ T stage } \\
\hline 0 & $2(3 \%)$ \\
\hline 1 & $21(27 \%)$ \\
\hline 2 & $20(26 \%)$ \\
\hline 3 & $11(14 \%)$ \\
\hline 4 & $23(30 \%)$ \\
\hline \multicolumn{2}{|l|}{$\mathrm{N}$ stage } \\
\hline 0 & $9(12 \%)$ \\
\hline 1 & $4(5 \%)$ \\
\hline 2 & $37(48 \%)$ \\
\hline 3 & $27(35 \%)$ \\
\hline \multicolumn{2}{|l|}{ Overall stage } \\
\hline IIIA & $36(47 \%)$ \\
\hline IIIB & $41(53 \%)$ \\
\hline Concurrent chemotherapy & $70(91 \%)$ \\
\hline
\end{tabular}

distant recurrence at one and two years was $42 \%$ and $58 \%$, respectively (Fig. 1, panels A and B). In the full 77 patient dataset, none of the pre-RT PET metrics were prognostic of overall survival or time to regional/distant recurrence on univariate Cox proportional hazards analysis (Table 3 and Supplementary Table 1). One pre-RT PET metric, MTV, was associated with time to local recurrence (Table 2). In the 72 patients without mid-RT early progression, higher midRT TLG and MTV were associated with increased risk of local recurrence ( $p=0.029$ and 0.030 , respectively), and higher ratio of mid-to pre-RT SUV max $_{\operatorname{mas}}$ wassociated with higher risk of regional/distant recurrence $(p=0.021)$.

We examined cut-points for the PET metrics that could group patients into high or low risk of local recurrence and regional/distant recurrence (Fig. 1, panels C and D). When grouped into quartiles, mid-RT TLG was not significantly associated with cumulative incidence of local recurrence by Gray's test ( $p=0.256$ ), but visually there appeared to be a lower local recurrence risk in the lowest quartile compared to the other three quartiles. When grouped into quartiles, ratio of mid- to pre-RT SUV $\mathrm{max}_{\max }$ was significantly associated with cumulative incidence of regional/distant recurrence $(p=0.044)$.

Five of seventy-seven mid-RT PET/CT scans showed clear evidence of early disease progression with appearance of new lesions, usually in out-of-field regional nodes. As there have been few reports on outcomes of patients with mid-RT imaging progression, we examined these patients in detail, along with two patients not included in the main analysis of 77 patients due to having been treated later than the inclusion cut-off and/or having stage II disease (Supplementary Table 2). In five of the seven patients, the radiation field was enlarged to include the new site of disease. The new site was treated to higher dose per fraction to ensure an effective tumoricidal dose but still finish radiation therapy within 6-8 weeks. An example of this approach is shown in Fig. 2. Except for the two patients treated recently with short follow-up, all of the patients with mid-RT progression are deceased. However, none of the five patients with field enlargement had further progression in the area of field enlargement.
Of the 72 patients without out-of-field progression on mid-RT PET/CT, 16 had an increase in one or more of the PET metrics on mid-RT PET/CT compared to pre-RT PET/CT. This most commonly occurred with mid-RT MTV, for which 12 patients had an increase compared to pre-RT PET/CT (median increase of $14.5 \%$, range 0.07 $325.6 \%$ ). There was no difference in outcomes between these 16 patients and the 56 patients with decrease in all PET metrics: for endpoints of overall survival, local recurrence, and regional/distant recurrence, $p$ values were $0.514-0.756$.

\section{Discussion}

We examined the prognostic value of pre- and mid-RT PET/CT metrics for patients with stage III NSCLC treated with definitive, modestly dose intensified RT, mostly with concurrent chemotherapy. We also analyzed outcomes for patients with early progression on mid-RT PET/CT. The overall outcomes in the cohort of 77 patients were similar to other recent reports in stage III patients treated with chemoRT, such as the RTOG 0617 multicenter trial [2]. Our patients' median overall survival was 23 months, similar to the RTOG 0617 median survival of around 24 months, and our patients' two-year cumulative incidence of local recurrence of $24 \%$ is less than the RTOG 0617 rate of around $34 \%$. One difference is that $53 \%$ of our patients had more advanced stage IIIB disease compared to $35 \%$ in RTOG 0617.

None of the PET metrics were associated with overall survival, but some were prognostic of local recurrence or regional/distant recurrence. Of the PET metrics, the statistically strongest findings were the correlation of higher mid-RT TLG with local recurrence $(p=0.03)$, and the correlation of higher ratio of mid- to pretreatment $\operatorname{SUV}_{\max }$ with regional/distant recurrence $(p=0.02)$. Interestingly, mid-RT GTV defined on CT without PET was also prognostic of local recurrence $(p=0.04)$, suggesting that CT alone also provides useful prognostic information. In a small number of patients, mid-RT PET/CT demonstrated early out-of-field progression most often in lymph nodes, and this was associated with subsequent distant progression. In 16 patients without out-of-field progression but with mid-RT increase in one or more of the PET metrics, outcomes were the same as for patients without mid-RT increase in PET metrics. This suggests that if FDG avidity of the treated lesions increases during treatment, this is more likely due to treatment-induced inflammation than progressive tumor and treatment should be continued.

There are several strengths to this analysis. As far as we are aware, this is the largest series examining prognostic value of mid-RT PET/CT in lung cancer. Also, we are not aware of a prior detailed analysis of outcomes of patients with progression on mid-RT PET/CT. We studied a uniform patient population by only including stage III patients treated with definitive chemoRT using consistent highly conformal techniques and modestly intensified RT dose. Almost all PET scans were done on two scanners within our department, ensuring technical consistency of PET metrics. The main limitation of the study is its retrospective nature. Also, as mid-RT PET/CT scans were not obtained as part of a research protocol, there was some variation in timing of PET/CT during the RT course.

Since pre-RT PET metrics such as MTV have been found to be associated with overall survival in several series that included stage III patients, we examined their prognostic value [19-22]. With respect to pre-RT PET metrics, our results closely parallel the findings of a secondary analysis of 230 patients on the ACRIN 6668/RTOG 0235 clinical trial by Bazan et al. [20]. In that analysis, higher pre-RT MTV was associated with worse overall survival, but this effect was diminished when the prescription dose exceeded $60 \mathrm{~Gy}$. In our current series of patients treated with modestly dose 
A

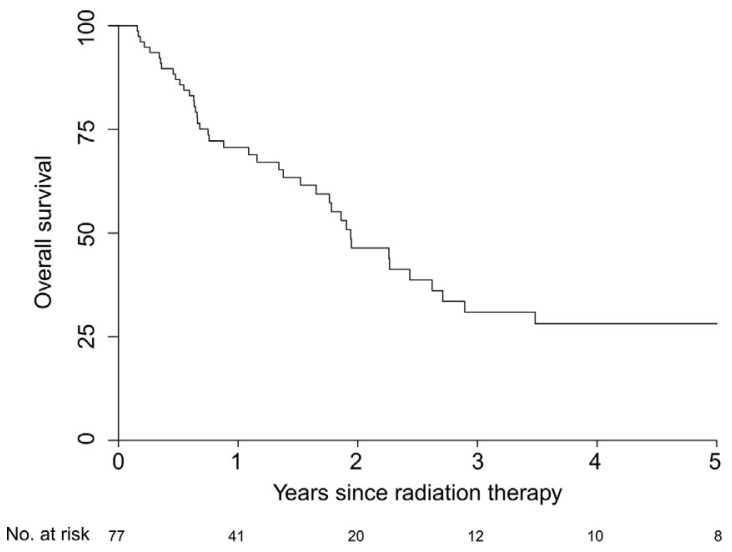

C

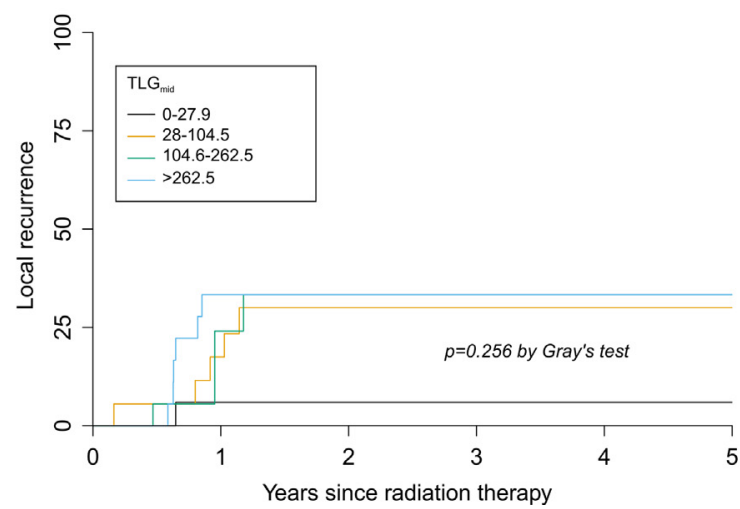

B

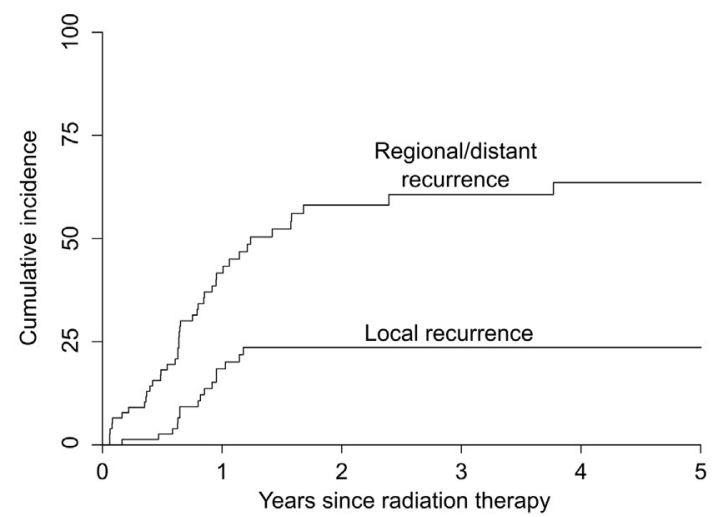

D

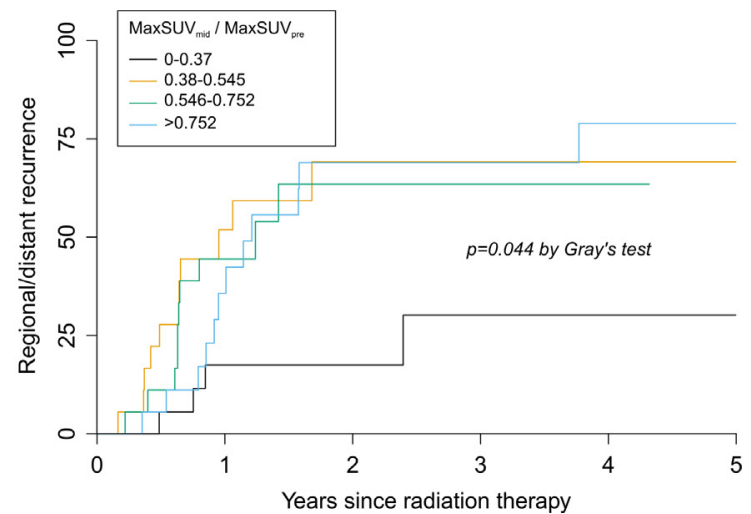

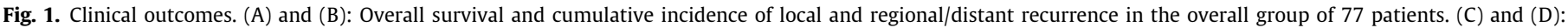

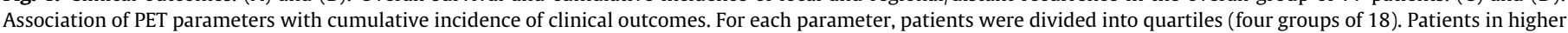

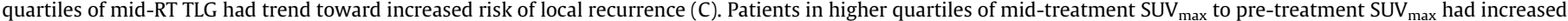
risk of regional/distant recurrence (D).

Table 2

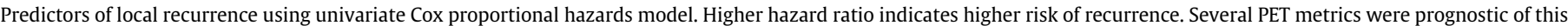
endpoint.

\begin{tabular}{|c|c|c|c|}
\hline & Variable & Hazard ratio $(95 \% \mathrm{CI})$ & $p$ value \\
\hline Clinical factors & $\begin{array}{l}\text { Karnofsky performance status (continuous variable) } \\
\text { Stage (IIIB vs. IIIA) }\end{array}$ & $\begin{array}{l}0.97(0.93-1.02) \\
1.04(0.39-2.78)\end{array}$ & $\begin{array}{l}0.195 \\
0.944\end{array}$ \\
\hline CT parameters & $\begin{array}{l}\text { GTV }_{\text {pre }}^{*} \\
\text { GTV }_{\text {mid }}^{*}\end{array}$ & $\begin{array}{l}1.58(0.90-2.77) \\
1.79(1.03-3.11)\end{array}$ & $\begin{array}{l}0.114 \\
0.039\end{array}$ \\
\hline Pre-RT PET parameters & $\begin{array}{l}\text { MTV }_{\text {pre }}{ }^{*} \\
\text { TLG }_{\text {pre }} \\
\text { MaxSUV }_{\text {pre }}\end{array}$ & $\begin{array}{l}1.53(1.00-2.33) \\
1.35(0.96-1.90) \\
1.02(0.97-1.07)\end{array}$ & $\begin{array}{l}\mathbf{0 . 0 4 9} \\
0.082 \\
0.406\end{array}$ \\
\hline Mid-RT PET parameters & $\begin{array}{l}\mathrm{MTV}_{\text {mid }} / \mathrm{MTV}_{\text {pre }} \\
\mathrm{TLG}_{\text {mid }} / \mathrm{TLG}_{\text {pre }} \\
\text { MaxSUV }_{\text {mid }} / \text { MaxSUV } \\
\text { MTV }_{\text {mid }} \\
\text { TLG }_{\text {mid }} \\
\text { MaxSUV }_{\text {mid }}\end{array}$ & $\begin{array}{l}1.22(0.51-2.92) \\
1.25(0.26-5.94) \\
0.87(0.13-5.72) \\
1.58(1.05-2.38) \\
1.57(1.05-2.34) \\
1.02(0.94-1.09)\end{array}$ & $\begin{array}{l}0.656 \\
0.779 \\
0.884 \\
\mathbf{0 . 0 3 0} \\
\mathbf{0 . 0 2 9} \\
0.675\end{array}$ \\
\hline
\end{tabular}

MTV = metabolic tumor volume.

TLG = total lesion glycolysis.

SUV $=$ standardized uptake value

Bold values indicate $p<0.05$.

Log transformed.

intensified RT, the prescription dose was higher than 60 Gy in $87 \%$ of patients. Furthermore, because we used IMRT with a volumetric prescription convention (with 95\% of the PTV receiving at least the prescription dose) and daily pre-treatment image guidance to ensure accurate delivery, the actual dose received by the PTV was even higher. Thus our lack of association between pre-RT MTV and overall survival is consistent with the secondary analysis of ACRIN 6668/RTOG 0235. With respect to local control, Bazan 
Table 3

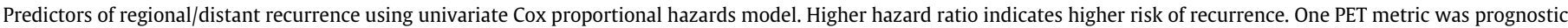
of this endpoint.

\begin{tabular}{|c|c|c|c|}
\hline & Variable & Hazard ratio $(95 \% \mathrm{CI})$ & $p$ value \\
\hline Clinical factors & $\begin{array}{l}\text { Karnofsky performance status (continuous variable) } \\
\text { Stage (IIIB vs. IIIA) }\end{array}$ & $\begin{array}{l}0.98(0.95-1.02) \\
1.10(0.60-2.03)\end{array}$ & $\begin{array}{l}0.339 \\
0.757\end{array}$ \\
\hline CT parameters & $\begin{array}{l}\mathrm{GTV}_{\text {pre }} \\
\text { GTV }_{\text {mid }}\end{array}$ & $\begin{array}{l}1.34(0.96-1.89) \\
1.25(0.91-1.72)\end{array}$ & $\begin{array}{l}0.089 \\
0.162\end{array}$ \\
\hline Pre-RT PET parameters & $\begin{array}{l}\text { MTV }_{\text {pre }}^{*} \\
\text { TLG }_{\text {pre }}^{*} \\
\text { MaxSUV }_{\text {pre }}\end{array}$ & $\begin{array}{l}1.20(0.95-1.52) \\
1.11(0.91-1.35) \\
0.99(0.96-1.03)\end{array}$ & $\begin{array}{l}0.121 \\
0.317 \\
0.665\end{array}$ \\
\hline Mid-RT PET parameters & $\begin{array}{l}\mathrm{MTV}_{\text {mid }} / \mathrm{MTV}_{\text {pre }} \\
\mathrm{TLG}_{\text {mid }} / \mathrm{TLG}_{\text {pre }} \\
\text { MaxSUV }_{\text {mid }} / M \text { MaxSUV }_{\text {pre }} \\
\text { MTV }_{\text {mid }} \\
\text { TLG }_{\text {mid }} \\
\text { MaxSUV }_{\text {mid }}\end{array}$ & $\begin{array}{l}1.17(0.67-2.05) \\
2.44(0.90-6.62) \\
4.6(1.26-16.76) \\
1.05(0.85-1.29) \\
1.13(0.92-1.38) \\
1.02(0.98-1.07)\end{array}$ & $\begin{array}{l}0.573 \\
0.080 \\
\mathbf{0 . 0 2 1} \\
0.655 \\
0.262 \\
0.344\end{array}$ \\
\hline
\end{tabular}

MTV = metabolic tumor volume

TLG $=$ total lesion glycolysis.

SUV $=$ standardized uptake value.

Bold values indicate $p<0.05$.

Log transformed.
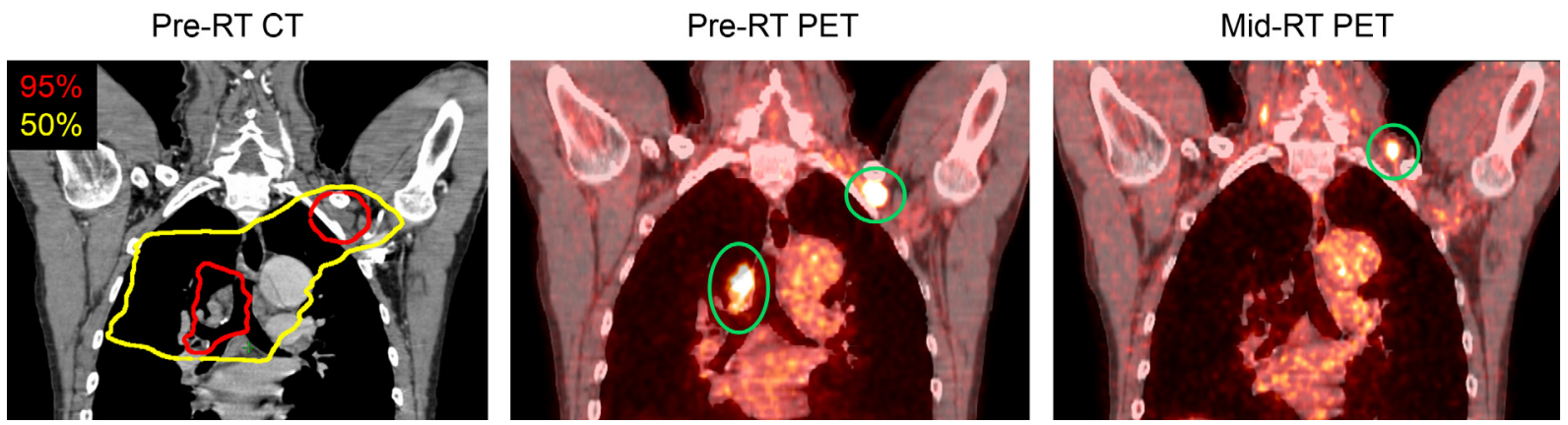

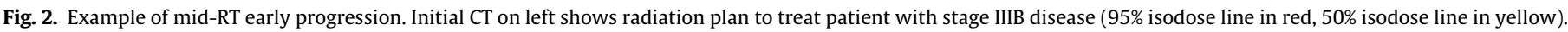

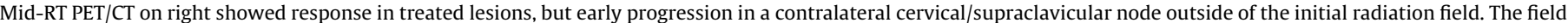

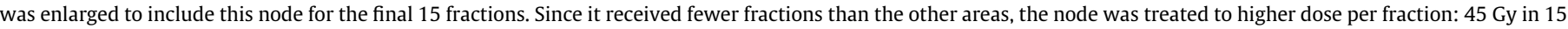

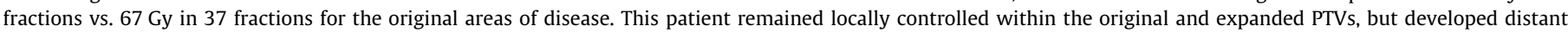
metastases and died 8 months after start of radiation. $\mathrm{RT}$ = radiation therapy

et al. found that higher pre-RT MTV was associated with higher local recurrence rate, an effect that appeared to be mitigated with higher RT dose. Similarly, our analysis found higher pre-RT MTV to be significantly associated with local recurrence.

Several other series, with sample sizes of 14-53 patients, have examined the prognostic value of mid-RT PET metrics in patients receiving radiation therapy for stage III NSCLC [8-12]. It is notable that each series identified different metrics as prognostic, ranging from SUV $_{\max }[8]$, to percentage decrease in MTV and mean SUV [9], percentage decrease in primary tumor mean SUV [11], and TLG [12]. As with the current series, most of these studies were retrospective with multiple endpoints examined, which raises statistical issues including multiple comparisons and selective reporting of endpoints. This makes it hard to draw firm conclusions about prognostic value of mid-RT PET metrics. In our series, higher mid-RT MTV and TLG were associated with local recurrence, suggesting that these metrics may help select patients (and possibly lesions) that would benefit from further local dose intensification. The ongoing multicenter RTOG 1106 study will provide additional prospective data that may help to clarify this issue [23]. Another promising direction is analyzing intratumoral heterogeneity of FDG uptake [24,25].

We identified seven patients (five from the seventy-seven in the main analysis, and two treated more recently) who had early disease progression identified on mid-RT PET/CT. By enlarging the radiation field and treating the area of progression to high dose per fraction, it was possible to control disease in the area of progression. However, five out of seven of these patients died from lung cancer or treatment side effects. This suggests that mid-RT out-of-field progression indicates resistance to chemotherapy and aggressive disease biology. Further investigation will be needed to determine whether additional salvage strategies, such as early switching of concurrent systemic therapy, would be beneficial.

In conclusion, for stage III NSCLC patients treated with modestly dose intensified highly conformal RT and chemotherapy, we found that some pre- and mid-RT PET metrics were prognostic of local recurrence and regional/distant recurrence, but not overall survival. The findings relating to local recurrence provide support for the ongoing RTOG 1106 trial and other trials examining adaptive radiation therapy, with higher dose given to areas of residual FDG uptake on mid-RT PET [23]. The optimal management of patients with early progression identified on mid-RT PET remains to be determined.

\section{Conflict of interest statement}

Dr. Gensheimer receives research support from Varian Medical Systems. Dr. Wakelee reports grants from AstraZeneca, personal fees from Peregrine, grants from Novartis, personal fees from ACEA, 
grants and personal fees from Pfizer, grants from BMS, grants from XCovery, grants from Celegene, grants from Roche/Genentech, grants from MedImmune, grants from Lilly, grants from Gilead, grants from Pharmacyclics, and personal fees from Helsinn. Dr. Neal reports personal fees from Clovis, personal fees from CARET/Physicians Resource Mgmt., grants and personal fees from Nektar, grants and personal fees from BI, grants from Genentech/Roche, grants from Merck, grants from Arqule, and grants from Exelixis. Dr. Loo receives research support from RaySearch Laboratories. He receives research support and educational lecture honoraria from Varian Medical Systems. He is a board member of TibaRay, Inc.

\section{Appendix A. Supplementary data}

Supplementary data associated with this article can be found, in the online version, at http://dx.doi.org/10.1016/j.radonc.2017.08. 007.

\section{References}

[1] Goldstraw P, Crowley J, Chansky K, et al. The IASLC lung cancer staging project: proposals for the revision of the TNM stage groupings in the forthcoming (seventh) edition of the TNM Classification of malignant tumours. J Thorac Oncol 2007;2:706-14.

[2] Bradley JD, Paulus R, Komaki R, et al. Standard-dose versus high-dose conformal radiotherapy with concurrent and consolidation carboplatin plus paclitaxel with or without cetuximab for patients with stage IIIA or IIIB nonsmall-cell lung cancer (RTOG 0617): a randomised, two-by-two factorial phase 3 study. Lancet Oncol 2015;16:187-99.

[3] De Ruysscher D, Wanders S, Minken A, et al. Effects of radiotherapy planning with a dedicated combined PET-CT-simulator of patients with non-small cell lung cancer on dose limiting normal tissues and radiation dose-escalation: a planning study. Radiother Oncol 2005;77:5-10.

[4] Konert T, Vogel W, MacManus MP, et al. PET/CT imaging for target volume delineation in curative intent radiotherapy of non-small cell lung cancer: IAEA consensus report 2014. Radiother Oncol 2015;116:27-34.

[5] Hallqvist A, Alverbratt C, Strandell A, et al. Positron emission tomography and computed tomographic imaging (PET/CT) for dose planning purposes of thoracic radiation with curative intent in lung cancer patients: a systematic review and meta-analysis. Radiother Oncol 2017:123:71-7.

[6] van Baardwijk A, Bosmans G, Dekker A, et al. Time trends in the maximal uptake of FDG on PET scan during thoracic radiotherapy. A prospective study in locally advanced non-small cell lung cancer (NSCLC) patients. Radiother Oncol 2007:82:145-52.

[7] Nygård L, Vogelius IR, Fischer BM, et al. Early lesion-specific (18)F-FDG PET response to chemotherapy predicts time to lesion progression in locally advanced non-small cell lung cancer. Radiother Oncol 2016;118:460-4.

[8] Vera P, Mezzani-Saillard S, Edet-Sanson A, et al. FDG PET during radiochemotherapy is predictive of outcome at 1 year in non-small-cell lung cancer patients: a prospective multicentre study (RTEP2). Eur J Nucl Med Mol Imaging 2014:41:1057-65.
[9] Huang W, Liu B, Fan M, et al. The early predictive value of a decrease of metabolic tumor volume in repeated (18)F-FDG PET/CT for recurrence of locally advanced non-small cell lung cancer with concurrent radiochemotherapy. Eur J Radiol 2015;84:482-8.

[10] Kong FM, Frey KA, Quint LE, et al. A pilot study of [18F]fluorodeoxyglucose positron emission tomography scans during and after radiation-based therapy in patients with non small-cell lung cancer. J Clin Oncol 2007;25:3116-23.

[11] van Elmpt W, Ollers M, Dingemans AM, Lambin P, De Ruysscher D. Response assessment using 18F-FDG PET early in the course of radiotherapy correlates with survival in advanced-stage non-small cell lung cancer. J Nucl Med 2012;53:1514-20.

[12] Yossi S, Krhili S, Muratet JP, Septans AL, Campion L, Denis F. Early assessment of metabolic response by 18F-FDG PET during concomitant radiochemotherapy of non-small cell lung carcinoma is associated with survival: a retrospective single-center study. Clin Nucl Med 2015;40:e215-21.

[13] Wahl RL, Jacene H, Kasamon Y, Lodge MA. From RECIST to PERCIST: evolving considerations for PET response criteria in solid tumors. J Nucl Med 2009;50:122S-50S.

[14] Radford J, Illidge T, Counsell N, et al. Results of a trial of PET-directed therapy for early-stage Hodgkin's lymphoma. N Engl J Med 2015;372:1598-607.

[15] Johnson P, Federico M, Kirkwood A, et al. Adapted treatment guided by interim PET-CT scan in advanced Hodgkin's lymphoma. $N$ Engl J Med 2016:374:2419-29.

[16] Mehanna H, Wong WL, McConkey CC, et al. PET-CT surveillance versus neck dissection in advanced head and neck cancer. $N$ Engl $J$ Med 2016;374:1444-54.

[17] Machtay M, Duan F, Siegel BA, et al. Prediction of survival by [18F] fluorodeoxyglucose positron emission tomography in patients with locally advanced non-small-cell lung cancer undergoing definitive chemoradiation therapy: results of the ACRIN 6668/RTOG 0235 trial. J Clin Oncol 2013;31:3823-30.

[18] Sridhar P, Mercier G, Tan J, Truong MT, Daly B, Subramaniam RM. FDG PET metabolic tumor volume segmentation and pathologic volume of primary human solid tumors. AJR Am J Roentgenol 2014;202:1114-9.

[19] Ohri N, Duan F, Machtay M, et al. Pretreatment FDG-PET metrics in stage III non-small cell lung cancer: ACRIN 6668/RTOG 0235. J Natl Cancer Inst 2015;107:djv004.

[20] Bazan JG, Duan F, Snyder BS, et al. Metabolic tumor volume predicts overall survival and local control in patients with stage III non-small cell lung cancer treated on ACRIN 6668/RTOG 0235. Eur J Nucl Med Mol Imaging 2016 [Epub ahead of print].

[21] Liao S, Penney BC, Wroblewski K, et al. Prognostic value of metabolic tumor burden on 18F-FDG PET in nonsurgical patients with non-small cell lung cancer. Eur J Nucl Med Mol Imaging 2012;392:27-38.

[22] Lee P, Bazan JG, Lavori PW, et al. Metabolic tumor volume is an independent prognostic factor in patients treated definitively for non-small-cell lung cancer. Clin Lung Cancer 2012;13:52-8.

[23] Kong, F.-M., RTOG 1106 protocol information. <https://www.rtog.org/ ClinicalTrials/ProtocolTable/StudyDetails.aspx?study=1106> (Updated Jan. 26, 2016. Accessed Sept. 28); 2016.

[24] Dong X, Sun X. Sun L, et al. Early change in metabolic tumor heterogeneity during chemoradiotherapy and its prognostic value for patients with locally advanced non-small cell lung cancer. PLoS One 2016;11:e0157836.

[25] Wu J, Gensheimer MF, Dong X, et al. Robust intratumor partitioning to identify high-risk subregions in lung cancer: a pilot study. Int J Radiat Oncol Biol Phys 2016;95:1504-12. 